\title{
Which Growth Charts for Today's Indian Children?
}

\author{
VAMAN KHADILKaR AND ANURADHa KHADILKaR \\ From Hirabai Cowasji Jehangir Medical Research Institute, Jehangir Hospital, 32, Sassoon Road, Pune 411001. \\ vamankhadilkar@gmail.com
}

A ssessment of children's growth using anthropometric parameters is crucial to study the nutritional status of a population, and is also useful in analyzing growth disorders. Reference data are crucial to growth monitoring and help health care professionals and policymakers to diagnose under-nutrition (stunting and wasting), overweight and obesity. Children's growth patterns change with time and references need to be updated regularly; this is especially true in a country like India which is in a phase of nutritional transition [1,2]. Indian Academy of Pediatrics (IAP) therefore revised National growth references in 2015 [3]. These charts are based on large nationwide data collected on middle and upper middle class children (33148, 18170 boys) over last decade. A need to field test the IAP 2015 charts was pointed out previously, so that their applicability across India can be validated [4].

An important strength of IAP 2015 charts is that they do not 'normalize' obesity. IAP 2015 BMI charts have been adjusted to 23 and 27 adult equivalent cut-offs as per the WHO recommendation for Asian Indians and these cut-offs are very close to the Asian cut-offs by International Obesity Task Force (IOTF) [5]. This has been shown by a recent study from Srinagar [6].

Since the inception of IAP 2015 charts, several studies across India have used them to assess prevalence of short stature, and overweight/obesity [6-8]. IAP 2015 charts detect more children with overweight/obesity than the WHO, CDC or Agarwal charts. Marginally higher percentage of children are detected as short by IAP 2015 charts as compared to Agarwal charts because IAP 2015 charts incorporate the secular trend in height.

In this issue of Indian Pediatrics, Singh, et al. [9] have compared IAP 2015, Agarwal 1992 and WHO 2007 references in children in a narrow age group of 8-15 years from one urban private and one government school from north Delhi. Other studies mentioned earlier are on slightly larger numbers; 2175 children by Lohiya, et al. [7], and 1500 children by Chudasma, et al. [10], covering ages from 5-18 years. The study by Singh, et al. [9] shows a good agreement between IAP 2015 and Agarwal charts in classifying subjects into categories of $\mathrm{BMI}(\mathrm{K}=0.82)$ and short stature $(\mathrm{K}=0.99)$. While this observation is valid, it is important to note the mean $\mathrm{Z}$ scores for height, weight and BMI are close to the IAP 2015 means as compared to the other charts, suggesting that urban Indian children's growth patterns are closest to the IAP 2015 reference standard [6-10].

Singh, et al. [9] also make a note of the secular trend in height in IAP 2015 charts as compared to the Agarwal charts (lower mean height for age $\mathrm{Z}$ scores in the IAP 2015). Similar data for weight and BMI are also presented by studies quoted in the earlier paragraphs. The observation by Singh, et al. [9] that IAP 2015 charts pick up more overweight and obese children than WHO and Agarwal charts has also been echoed by previous studies $[6,7,10]$.

As IAP 2015 charts will be put to use more often in future in epidemiological studies as well as disease situations, their strength and limitations will be revealed further. Thus, articles such as the one by Singh, et al. [9] will help to improve and update future growth references for Indian children.

Funding: None; Competing interests: None stated.

\section{REFERENCES}

1. Buckler JMH. Growth disorders in Children. 1st ed. London: BMJ Publishing Group; 1994.

2. Rao S. Nutritional status of the Indian population. J Biosci. 2001; 26: 481-9.

3. Indian Academy of Pediatrics Growth Charts Committee, Khadilkar V, Yadav S, Agrawal KK, Tamboli S, Banerjee $\mathrm{M}$, Cherian A, et al. Revised IAP growth charts for height, weight and body mass index for 5- to 18-year-old Indian children. Indian Pediatr. 2015;52:47-55.

4. Bajpai A. Coming of age of IAP 2015 growth charts: The way forward. Indian J Pediatr.2018;85:715.

5. Cole TJ, Bellizzi MC, Flegal KM, Dietz WH. Establishing a standard definition for child overweight and obesity worldwide: International survey. BMJ. 2000;320: 1240-3. 
EDITORIAL

6. Haq I, Raja M, Ahmad M. A comparison of the 2015 Indian Academy of Pediatrics, International Obesity Task Force and World Health Organization growth references among 518-year-old children. Ann Trop Med Public Health. 2017;10:1814-9.

7. Lohiya N, Khadilkar V, Pawar S, Khadilkar A, Chiplonkar S, Jahagirdar R. Field testing of IAP 2015 charts. Indian J Pediatr. 2018;85:723-728.

8. Patil A, Patil P, Bhandarkar P. Adolescent obesity by different growth charts and its efficacy with central adiposity among school-age children in Mumbai. International Journal of Population Studies 2019; 4:35-42.

9. Singh P, Gandhi S, Malhotra R, Seth A. Impact of using different growth references on interpretation of anthropometric parameters of children aged 8-15 years. Indian Pediatr. 2020;57:124-8.

10. Chudasama RK, Eshwar T, Thakrar D, Eshwar ST. Prevalence of obesity and overweight among school children aged 8-18 years in Rajkot, Gujarat. Indian Pediatr. 2016;53:743-4. 